\title{
Convergent Synthesis of the E'FGH' ring fragment of ciguatoxin 1B via an acetylene cobalt complex strategy
}

\author{
Shigeyuki Takai and Minoru Isobe* \\ Laboratory of Organic Chemistry, School of Bioagricultural Sciences, Nagoya University \\ Chikusa, Nagoya 464-8601, Japan
}

\section{Supporting Information}

\section{Iodide 7}

Iodide 7. IR (KBr) $v_{\max } 2955,2930,2857,1472,1463,1362,1253,1203,838,776$ $\mathrm{cm}^{-1}$. ${ }^{1} \mathrm{H} \mathrm{NMR}\left(\mathrm{CDCl}_{3}, 300 \mathrm{MHz}\right) \delta 0.09$ (3H, s, TBS), $0.12(3 \mathrm{H}, \mathrm{s}, \mathrm{TBS}), 0.88(9 \mathrm{H}$, s, tBu $, 1.49(1 \mathrm{H}, \mathrm{m}, \mathrm{H}-3), 1.66(2 \mathrm{H}, \mathrm{m}, \mathrm{H}-2), 2.03(1 \mathrm{H}, \mathrm{m}, \mathrm{H}-3), 2.82(1 \mathrm{H}$, ddd $J=$ 8.5, 6.0, 3.0 Hz, H-5), 3.33 (1H, dd, $J=10.5,6.0 \mathrm{~Hz}, \mathrm{H}-6), 3.41$ (1H, m, H-4), 3.43 $(1 \mathrm{H}, \mathrm{ddd}, J=11.5,7.5,3.5 \mathrm{~Hz}, \mathrm{H}-1), 3.51(1 \mathrm{H}, \mathrm{dd}, J=10.5,3.0 \mathrm{~Hz}, \mathrm{H}-6), 3.97$ $(1 \mathrm{H}, \mathrm{dm}, J=11.5 \mathrm{~Hz}, \mathrm{H}-1)$. ${ }^{13} \mathrm{C} \mathrm{NMR}\left(\mathrm{CDCl}_{3}, 75 \mathrm{MHz}\right) \delta-4.64,-4.00,9.23,17.78$, 25.39, 25.68, 33.03, 67.94, 71.16, 80.78. ESI Q-TOF MS calcd for $\mathrm{C}_{12} \mathrm{H}_{25} \mathrm{INaO}_{2} \mathrm{Si}$ $[\mathrm{M}+\mathrm{Na}]^{+} 379.057$, found 379.065. $[\alpha]_{\mathrm{D}}^{27}+59.3^{\circ}\left(c 0.86, \mathrm{CHCl}_{3}\right)$. Anal. Calcd for $\mathrm{C}_{12} \mathrm{H}_{25} \mathrm{IO}_{2} \mathrm{Si}: \mathrm{C}, 40.45 ; \mathrm{H}, 7.07$. Found: C, 40.45; H, 7.22.

\section{Nitrile 8}

To a solution of iodide 7 (3.38 g, $9.49 \mathrm{mmol})$ in dimethylsulfoxide $(47 \mathrm{ml})$ was added sodium cyanide (697 mg, $14.2 \mathrm{mmol}$ ). After stirring for $2 \mathrm{~h}$ at $80{ }^{\circ} \mathrm{C}$ under nitrogen atmosphere, the reaction mixture was poured into ammonium chloride aq. at $\mathrm{rt}$ in draft and then extracted with ether (x 3). The combined organic layer was washed with brine and dried over anhydrous sodium sulfate. Concentration of the solvent gave a crude oil, which was purified by silica gel column chromatography (ethyl acetate / hexane $=10$ / 90) to afford nitrile $8(2.31 \mathrm{~g}, 95 \%)$ as a colorless oil.

Nitrile 8. IR (KBr) $v_{\max } 2955,2931,2859,2251,1472,1363,1254,1134,1127$, 1101, 1050, 869, 839, 778, $670 \mathrm{~cm}^{-1}$. 1 $\mathrm{H} \mathrm{NMR}\left(\mathrm{CDCl}_{3}, 400 \mathrm{MHz}\right) \delta 0.09(3 \mathrm{H}, \mathrm{s}$, TBS), 0.11 (3H, s, TBS), $0.88\left(9 \mathrm{H}, \mathrm{s},{ }^{\mathrm{t}} \mathrm{Bu}\right), 1.45(1 \mathrm{H}, \mathrm{dddd}, J=12.5,12.5,10.5,4.5$ $\mathrm{Hz}, \mathrm{H}-3), 1.60-1.79$ (2H, m, H-2), 2.02-2.10 (1H, dm, J=12.5 Hz, H-3), $2.58(1 \mathrm{H}$, 
dd, $J=16.5,6.5 \mathrm{~Hz}, \mathrm{H}-6), 2.73(1 \mathrm{H}, \mathrm{dd}, J=16.5,3.5 \mathrm{~Hz}, \mathrm{H}-6), 3.26(1 \mathrm{H}$, ddd $J=$ 9.0, 6.5, $3.5 \mathrm{~Hz}, \mathrm{H}-5), 3.36$ (1H, ddd, $J=11.5,11.5,3.5 \mathrm{~Hz}, \mathrm{H}-1), 3.44$ (1H, ddd, $J$ $=10.5,9.0,4.5 \mathrm{~Hz}, \mathrm{H}-4), 3.94(1 \mathrm{H}$, dddd, $J=11.5,4.5,2.0,1.5 \mathrm{~Hz}, \mathrm{H}-1) .{ }^{13 \mathrm{C}}$ NMR $\left(\mathrm{CDCl}_{3}, 100 \mathrm{MHz}\right) \delta-4.89,-3.97,17.79,21.40,25.14,25.66,33.44,67.98$, 70.14, 77.91, 117.51. ESI Q-TOF MS calcd for $\mathrm{C}_{13} \mathrm{H}_{25} \mathrm{NNaO}_{2} \mathrm{Si}[\mathrm{M}+\mathrm{Na}]^{+} 278.155$, found 278.152. $[\alpha]_{\mathrm{D}} 27+57.2^{\circ}\left(c\right.$ 1.78, $\left.\mathrm{CHCl}_{3}\right)$. Anal. Calcd for $\mathrm{C}_{13} \mathrm{H}_{25} \mathrm{NO}_{2} \mathrm{Si}: \mathrm{C}$, 61.13; H, 9.87; N, 5.48. Found: C, 60.96; H, 10.25; N, 5.61.

\section{Aldehyde 9}

To a solution of nitrile $\mathbf{8}(1.42 \mathrm{~g}, 5.56 \mathrm{mmol})$ in dry toluene $(28 \mathrm{ml})$ was added dropwise a solution of $1.0 \mathrm{M}$ diisobutylaluminum hydride in hexane $(7.23 \mathrm{ml}, 7.23$ $\mathrm{mmol}$ ) at $-78{ }^{\circ} \mathrm{C}$ under nitrogen atmosphere. After stirring for $1 \mathrm{~h}$ at $-78{ }^{\circ} \mathrm{C}$, the reaction mixture was added $10 \%$ acetic acid aq. $(28 \mathrm{ml})$ at $-78{ }^{\circ} \mathrm{C}$. After warming up to $\mathrm{rt}$ gradually, the reaction mixture was added ether $(200 \mathrm{ml})$ and poured into a mixture of saturated sodium hydrogen carbonate aq. and saturated potassium sodium tartrate aq. (50 $\mathrm{ml} / 50 \mathrm{ml}$ ). After stirring for $1 \mathrm{~h}$ at $\mathrm{rt}$, the reaction mixture was extracted with ether (x 3 ). The combined organic layer was washed with brine and then dried over anhydrous sodium sulfate. Concentration of the solvent gave a crude oil, which was purified by silica gel column chromatography (ether / hexane $=10 / 90)$ to afford aldehyde 9 (836 $\mathrm{mg}, 58 \%)$ as a colorless oil

Aldehyde 9. IR (KBr) $v_{\max }$ 2956, 2931, 2858, 2728, 1731, 1473, 1363, 1260, 1129, $1101,838,777,670 \mathrm{~cm}^{-1}$. 1H NMR $\left(\mathrm{CDCl}_{3}, 300 \mathrm{MHz}\right) \delta 0.04(3 \mathrm{H}, \mathrm{s}, \mathrm{TBS}), 0.05$ $(3 \mathrm{H}, \mathrm{s}, \mathrm{TBS}), 0.86\left(9 \mathrm{H}, \mathrm{s},{ }^{\mathrm{t}} \mathrm{Bu}\right), 1.38-1.52(1 \mathrm{H}, \mathrm{m}, \mathrm{H}-3), 1.61-1.72(2 \mathrm{H}, \mathrm{m}, \mathrm{H}-2)$, 1.98-2.08 (1H, m, H-3), 2.42 (1H, ddd, $J=16.5,9.0,3.0 \mathrm{~Hz}, \mathrm{H}-6), 2.77$ (1H, ddd, $J$ $=16.5,4.0,2.0 \mathrm{~Hz}, \mathrm{H}-6), 3.28-3.40(1 \mathrm{H}, \mathrm{m}, \mathrm{H}-1$ and $\mathrm{H}-4), 3.58(1 \mathrm{H}, \mathrm{td}, J=9.0,4.0$ Hz, H-5), $3.85(1 \mathrm{H}, \mathrm{dm}, J=11.5 \mathrm{~Hz}, \mathrm{H}-1), 9.76(1 \mathrm{H}, \mathrm{dd}, J=3.0,2.0 \mathrm{~Hz},-\mathrm{CHO})$. ${ }_{13} \mathrm{C}$ NMR $\left(\mathrm{CDCl}_{3}, 75 \mathrm{MHz}\right) \delta-4.90,-4.11,17.77,25.39,25.64,33.32,46.70,67.81$, 70.98, 78.19, 201.84. ESI Q-TOF MS calcd for $\mathrm{C}_{13} \mathrm{H}_{26} \mathrm{NaO}_{3} \mathrm{Si}[\mathrm{M}+\mathrm{Na}]^{+}$281.155, found 281.161. $\left.[\alpha]_{\mathrm{D}}{ }^{26}+43.4^{\circ}(c) 0.85, \mathrm{CHCl}_{3}\right)$. Anal. Calcd for $\mathrm{C}_{13} \mathrm{H}_{26} \mathrm{O}_{3} \mathrm{Si}: \mathrm{C}$, 60.42; H, 10.14. Found: C, 60.36; H, 10.24.

\section{Silyl Enyne 11}

To a solution of 1,3-bis(triisopropylsilyl)propyne $\mathbf{1 0}(1.41 \mathrm{~g}, 4.00 \mathrm{mmol})$ in dist. tetrahydrofuran $(15 \mathrm{ml})$ was added dropwise a solution of $1.50 \mathrm{M} n$-BuLi in hexane 
$(2.45 \mathrm{ml}, 3.68 \mathrm{mmol})$ at $-78{ }^{\circ} \mathrm{C}$ under nitrogen atmosphere. After stirring for $20 \mathrm{~min}$ at 0 ${ }^{\circ} \mathrm{C}$, the reaction mixture was added dropwise a solution of aldehyde 9 (792 $\mathrm{mg}, 3.06$ mmol) in dist. tetrahydrofuran $(5 \mathrm{ml})$ at $-78{ }^{\circ} \mathrm{C}$. After stirring for $2 \mathrm{~h}$ at $-78{ }^{\circ} \mathrm{C}$, the reaction mixture was poured into saturated ammonium chloride aq. at $0{ }^{\circ} \mathrm{C}$ and then extracted with ether (x 3). The combined organic layer was washed with brine and dried over anhydrous sodium sulfate. Concentration of the solvent gave a crude oil, which was purified by silica gel column chromatography (ether / hexane $=2 / 98$ ) to afford silyl enyne $11(983 \mathrm{mg}, 73 \%, Z: E=4.9: 1)$ as a colorless oil.

Silyl Enyne 11 ( $Z$-isomer). IR (KBr) $v_{\max }$ 2943, 2892, 2866, 2148, 1464, 1252 , 1130, 1100, 1036, 999, 883, 838, 776, $677 \mathrm{~cm}^{-1}$. 1H NMR $\left(\mathrm{CDCl}_{3}, 400 \mathrm{MHz}\right) \delta 0.06$ (6H, s, TBS), 0.88 (9H, s, $\left.{ }^{\mathrm{B}} \mathrm{Bu}-\mathrm{TBS}\right), 1.08$ (18H, s, TIPS), 1.09 (3H, s, TIPS), 1.43 $(1 \mathrm{H}, \mathrm{dddd}, J=16.0,12.5,6.5,5.5 \mathrm{~Hz}, \mathrm{H}-3), 1.63(2 \mathrm{H}, \mathrm{m}, \mathrm{H}-2), 2.00(1 \mathrm{H}, \mathrm{dm}, J=$ $12.5 \mathrm{~Hz}, \mathrm{H}-3), 2.52$ (1H, dddd, $J=16.5,8.5,7.5,1.5 \mathrm{~Hz}, \mathrm{H}-6), 2.79$ (1H, dddd, $J=$ 16.5, 6.0, 3.5, $2.0 \mathrm{~Hz}, \mathrm{H}-6), 3.18(1 \mathrm{H}, \mathrm{ddd}, J=8.5,8.5,3.5 \mathrm{~Hz}, \mathrm{H}-5), 3.31(1 \mathrm{H}, \mathrm{m}$, $\mathrm{H}-1), 3.31(1 \mathrm{H}, \mathrm{m}, \mathrm{H}-4), 3.87(1 \mathrm{H}, \mathrm{dm}, J=11.5 \mathrm{~Hz}, \mathrm{H}-1), 5.62(1 \mathrm{H}$, ddd, $J=11.0$,

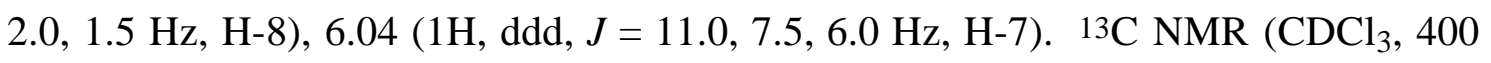
$\mathrm{MHz}) \delta-4.63,-4.11,11.33,17.95,18.68,25.64,25.85,33.38,33.61,67.80,71.46$, $81.81,95.41,103.91,110.85,141.56 .[\alpha]_{\mathrm{D}}^{22}+28.0^{\circ}\left(c 0.60, \mathrm{CHCl}_{3}\right)$. ESI Q-TOF MS calcd for $\mathrm{C}_{25} \mathrm{H}_{49} \mathrm{O}_{2} \mathrm{Si}_{2}[\mathrm{M}+\mathrm{H}]+437.327$, found 437.338.

\section{$E^{\prime}$ ring-enyne 12}

To a solution of silyl enyne $11(865 \mathrm{mg}, 1.98 \mathrm{mmol})$ in tetrahydrofuran $(10 \mathrm{ml})$ was added a solution of 1.0 M tetrabutylammonium fluoride in tetrahydrofuran $(15.8 \mathrm{ml}$, $15.8 \mathrm{mmol}$ ) at $0{ }^{\circ} \mathrm{C}$. After stirring for $10 \mathrm{~h}$ at $\mathrm{rt}$ under nitrogen atmosphere, the reaction mixture was poured into saturated ammonium chloride aq. at $0{ }^{\circ} \mathrm{C}$ and then extracted with ether (x 3). The combined organic layer was washed with brine and dried over anhydrous sodium sulfate. Concentration of the solvent gave a crude oil, which was purified by silica gel column chromatography (ether / hexane $=50 / 50)$ to afford an alcohol (308 $\mathrm{mg}$, 94\%) as a colorless oil.

To a solution of the alcohol $(98 \mathrm{mg}, 0.59 \mathrm{mmol})$ in dichloromethane $(3 \mathrm{ml})$ was added ethyl vinyl ether $(0.56 \mathrm{ml}, 5.90 \mathrm{mmol})$ and pyridinium $p$-toluenesulfonate $(44 \mathrm{mg}$, $0.18 \mathrm{mmol}$ ). After stirring for $2 \mathrm{~h}$ at $\mathrm{rt}$ under nitrogen atmosphere, the reaction mixture was poured into saturated sodium hydrogen carbonate aq. at $0{ }^{\circ} \mathrm{C}$ and then extracted with dichloromethane (x 3). The combined organic layer was washed with brine and dried over anhydrous sodium sulfate. Concentration of the solvent gave a crude oil, which was 
purified by silica gel column chromatography (ether / hexane $=5$ / 95) to afford E' ringenyne $12(137 \mathrm{mg}, 97 \%)$ as a colorless oil.

E' ring-enyne 12 (Z-isomer). IR (KBr) $v_{\max }$ 3290, 2977, 2939, 2853, 1441, 1395, $1378,1341,1280,1210,1131,1099,1059,1046,949,639 \mathrm{~cm}^{-1} .1 \mathrm{H} \mathrm{NMR}\left(\mathrm{CDCl}_{3}\right.$, $300 \mathrm{MHz}) \delta 1.20(3 \mathrm{H}, \mathrm{t}, J=7.0 \mathrm{~Hz}, \mathrm{EE}), 1.31(1 / 2 \times 3 \mathrm{H}, \mathrm{d}, J=5.5 \mathrm{~Hz}, \mathrm{EE}), 1.33$ $(1 / 2 \times 3 \mathrm{H}, \mathrm{d}, J=5.5 \mathrm{~Hz}, \mathrm{EE}), 1.34-1.56(1 \mathrm{H}, \mathrm{m}, \mathrm{H}-3), 1.60-1.72(2 \mathrm{H}, \mathrm{m}, \mathrm{H}-2), 2.14-$ 2.30 (1H, m, H-3), 2.44-2.82 (2H, m, H-6), 3.08-3.11 (1H, m, acetylenic), 3.14-3.42 (3H, m, H-1,H-4 and H-5), 3.43-3.72 (2H, m, EE), 3.89 (1H, dm, $J=12 \mathrm{~Hz}, \mathrm{H}-1)$, $4.75(1 / 2 \times 1 \mathrm{H}, \mathrm{q}, J=5.5 \mathrm{~Hz}, \mathrm{EE}), 4.84(1 / 2 \times 1 \mathrm{H}, \mathrm{q}, J=5.5 \mathrm{~Hz}, \mathrm{EE}), 5.55(1 \mathrm{H}, \mathrm{dm}, J=$ $11.0 \mathrm{~Hz}, \mathrm{H}-8), 6.19(1 \mathrm{H}, \mathrm{dm}, J=11.0 \mathrm{~Hz}, \mathrm{H}-7)$. ESI Q-TOF MS calcd for $\mathrm{C}_{14} \mathrm{H}_{23} \mathrm{O}_{3}$ $[\mathrm{M}+\mathrm{H}]+239.165$, found 239.177 .

\section{Propargyl Alcohol 16}

To a solution of silylacetylene $\mathbf{1 5}(620 \mathrm{mg}, 1.22 \mathrm{mmol})$ in methanol $(6 \mathrm{ml})$ was added $\mathrm{K}_{2} \mathrm{CO}_{3}(34 \mathrm{mg}, 0.24 \mathrm{mmol}$ ). After stirring for $2 \mathrm{~h}$ at $\mathrm{rt}$, the reaction mixture was poured into saturated ammonium chloride aq. at $0{ }^{\circ} \mathrm{C}$ and then extracted with ether (x 3). The combined organic layer was washed with brine and dried over anhydrous sodium sulfate. Concentration of the solvent gave a crude oil, which was purified by silica gel column chromatography (ethyl acetate / hexane $=20 / 80)$ to afford an alcohol $(475 \mathrm{mg}$, $99 \%)$.

To a solution of the alcohol $(17.8 \mathrm{~g}, 45.3 \mathrm{mmol})$ in benzylchloride $(110 \mathrm{ml})$ was added $\mathrm{KOH}$ powder $(12.7 \mathrm{~g}, 226.5 \mathrm{mmol})$. After stirring for $2 \mathrm{~h}$ at $90^{\circ} \mathrm{C}$, the reaction mixture was poured into saturated ammonium chloride aq. at $0{ }^{\circ} \mathrm{C}$ slowly and then extracted with ether (x 3). The combined organic layer was washed with brine and dried over anhydrous sodium sulfate. Concentration of the solvent gave a crude oil, which was purified by silica gel column chromatography (ether / hexane $=6 / 94$ ) to afford a benzyl ether $(19.1 \mathrm{~g}, 87 \%)$ as a pale yellow oil.

To a solution of the benzyl ether $(480 \mathrm{mg}, 0.99 \mathrm{mmol})$, which was treatment with toluene azeotrope in advance, in dist. tetrahydrofuran $(10 \mathrm{ml})$ was added a solution of $n$ BuLi (1.60 $\mathrm{M}$ in Hexane, $0.75 \mathrm{ml}, 1.19 \mathrm{mmol})$ at $-78{ }^{\circ} \mathrm{C}$ under nitrogen atmosphere. After stirring for $20 \mathrm{~min}$ at $0{ }^{\circ} \mathrm{C}$, a solution of $(\mathrm{HCHO})_{\mathrm{n}}(149 \mathrm{mg}, 4.96 \mathrm{mmol})$ in dist. tetrahydrofuran $5 \mathrm{ml}$ was added. After stirring for $1 \mathrm{~h}$ at $-78^{\circ} \mathrm{C}$ and for $16 \mathrm{~h}$ at $0{ }^{\circ} \mathrm{C}$, the reaction mixture was poured into saturated ammonium chloride aq. at $0{ }^{\circ} \mathrm{C}$ and then extracted with ether (x 3). The combined organic layer was washed with brine and dried over anhydrous sodium sulfate. Concentration of the solvent gave a crude oil, which was purified by silica gel column chromatography (ether / hexane $=5 / 95$ to $25 / 75$ ) to afford 
SM (76 mg, 16\%), formated-16 (84 mg, 16\%) and propargyl alcohol 16 (283 mg, $55 \%)$ as a pale yellow oil.

To a solution of formated-16 $(84 \mathrm{mg}, 0.16 \mathrm{mmol})$ in $\mathrm{MeOH}(3 \mathrm{ml})$ was added $\mathrm{K}_{2} \mathrm{CO}_{3}$ (22 $\mathrm{mg}, 0.16 \mathrm{mmol}$ ). After stirring for $20 \mathrm{~min}$ at $\mathrm{rt}$, the reaction mixture was poured into saturated ammonium chloride aq. at $0{ }^{\circ} \mathrm{C}$ and then extracted with ether (x 3$)$. The combined organic layer was washed with brine and dried over anhydrous sodium sulfate. Concentration of the solvent gave a crude oil, which was purified by silica gel column chromatography (ether / hexane $=50 / 50)$ to afford propargyl alcohol 16 (80 $\mathrm{mg}, 100 \%)$ as a pale yellow oil.

Propargyl Alcohol 16. IR (KBr) $v_{\max }$ 3434, 3071, 3047, 2958, 2932, 2892, 2858, 1962, 1895, 1830, 1590, 1473, 1428, 1390, 1364, 1304, 1158, 1113, 1028, 822, 742, 701, 613, $513 \mathrm{~cm}^{-1}$. ${ }^{1} \mathrm{H} \mathrm{NMR}\left(\mathrm{CDCl}_{3}, 400 \mathrm{MHz}\right) \delta 1.08\left(9 \mathrm{H}, \mathrm{s},{ }^{t} \mathrm{Bu}\right), 2.16(1 \mathrm{H}, \mathrm{br}-\mathrm{s}$, $-\mathrm{OH}), 3.37$ (1H, dd $J=10.0,5.5 \mathrm{~Hz}, \mathrm{H}-6), 3.47$ (1H, dd, $J=10.0,6.0 \mathrm{~Hz}, \mathrm{H}-6), 4.06$ $(2 \mathrm{H}, \mathrm{br}-\mathrm{d}, J=6.0 \mathrm{~Hz}$, propargylic), $4.26(1 \mathrm{H}, \mathrm{dm}, J=8.5 \mathrm{~Hz}, \mathrm{H}-1), 4.32-4.38(2 \mathrm{H}$, $\mathrm{m}, \mathrm{H}-2$ and $\mathrm{H}-5), 4.48\left(1 \mathrm{H}, \mathrm{d}, J=12.0 \mathrm{~Hz},-\mathrm{OCH}_{2} \mathrm{Ph}\right), 4.53(1 \mathrm{H}, \mathrm{d}, J=12.0 \mathrm{~Hz}$,

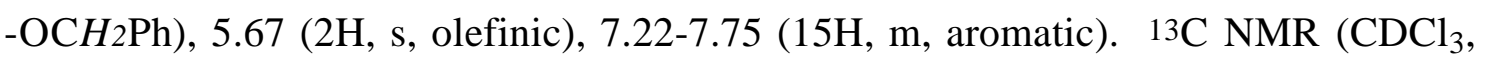
$100 \mathrm{MHz}) \delta 19.32,26.87,51.08,69.00,70.19,72.03,73.47,74.46,84.04,84.43$, $127.42,127.67,127.77,128.34,129.74,129.84,130.26,133.48,133.62,136.05$, 136.16, 137.92. ESI Q-TOF MS calcd for $\mathrm{C}_{32} \mathrm{H}_{36} \mathrm{NaO}_{4} \mathrm{Si}[\mathrm{M}+\mathrm{Na}]^{+} 535.228$, found 535.228. $[\alpha]_{\mathrm{D}}{ }^{24}-53.3^{\circ}\left(c 1.10, \mathrm{CHCl}_{3}\right)$. Anal. Calcd for $\mathrm{C}_{32} \mathrm{H}_{36} \mathrm{O}_{4} \mathrm{Si}: \mathrm{C}, 74.96 ; \mathrm{H}$, 7.08. Found: C, 74.75; H, 7.38.

\section{Allyl Alcohol 17}

To a solution of $\mathbf{1 6}(363 \mathrm{mg}, 0.71 \mathrm{mmol})$ in dist. THF $(7 \mathrm{ml})$ was added a solution of Red-Al ${ }^{\circledR}(65 \%$ in toluene, $0.43 \mathrm{ml}, 1.42 \mathrm{mmol})$ at $0{ }^{\circ} \mathrm{C}$. After stirring for $20 \mathrm{~min}$ at 0 ${ }^{\circ} \mathrm{C}$ and for $15 \mathrm{~min}$ at $\mathrm{rt}, 10 \%$ acetic acid aq. was added at $0{ }^{\circ} \mathrm{C}$ and then the reaction mixture was poured into a mixture of saturated sodium hydrogen carbonate aq. and saturated potassium sodium tartrate aq. (1vol./1vol.) at $0{ }^{\circ} \mathrm{C}$. After stirring for $1 \mathrm{~h} \mathrm{at} \mathrm{rt}$, the reaction mixture was extracted with ether (x 3). The combined organic layer was washed with water and dried over anhydrous sodium sulfate. Concentration of the solvent gave a crude oil, which was purified by silica gel column chromatography ( ether / hexane $=30 / 70)$ to afford allyl alcohol $17(344 \mathrm{mg}, 0.67 \mathrm{mmol}, 95 \%)$ as a colorless oil.

Allyl alcohol 17. IR (KBr) $v_{\max }$ 3448, 3071, 3032, 2931, 2858, 1473, 1428, 1390, 1363, 1310, 1111, 1091, 978, 862, 822, 741, 702, 612, $508 \mathrm{~cm}^{-1} .{ }^{1} \mathrm{H} \mathrm{NMR}\left(\mathrm{CDCl}_{3}\right.$, 
$300 \mathrm{MHz}) \delta 1.05\left(9 \mathrm{H}, \mathrm{s},{ }^{t} \mathrm{Bu}\right), 1.26(1 \mathrm{H}, \mathrm{br}-\mathrm{s},-\mathrm{OH}), 3.39(1 \mathrm{H}, \mathrm{dd} J=10.0,5.0 \mathrm{~Hz}$, H-6), 3.45 (1H, dd, $J=10.0,6.0 \mathrm{~Hz}, \mathrm{H}-6), 3.92$ (1H, ddd, $J=8.5,6.5,1.0 \mathrm{~Hz}, \mathrm{H}-1)$, $3.99\left(2 \mathrm{H}, \mathrm{br}-\mathrm{m}, \mathrm{H}-3^{\prime}\right), 4.17$ (1H, dddd, $\left.J=8.5,3.0,1.5,1.5 \mathrm{~Hz}, \mathrm{H}-2\right), 4.35(1 \mathrm{H}, \mathrm{m}$, $\mathrm{H}-5), 4.51\left(1 \mathrm{H}, \mathrm{d}, J=12.0 \mathrm{~Hz},-\mathrm{OCH} H_{2} \mathrm{Ph}\right), 4.55(1 \mathrm{H}, \mathrm{d}, J=12.0 \mathrm{~Hz},-\mathrm{OCH} \mathrm{Ph}), 5.56$ $\left(1 \mathrm{H}, \mathrm{ddt}, J=15.5,6.5,1.5 \mathrm{~Hz}, \mathrm{H}-1^{\prime}\right), 5.66(1 \mathrm{H}$, ddd, $J=10.5,1.5,1.5 \mathrm{~Hz}$, cisolefinic), 5.75 (1H, ddd, $J=10.5,2.0,1.5 \mathrm{~Hz}$, cis-olefinic), 5.91 ( $1 \mathrm{H}, \mathrm{dtd}, J=15.5$, 5.5, $1.0 \mathrm{~Hz}, \mathrm{H}-2 '), 7.22-7.69\left(15 \mathrm{H}, \mathrm{m}\right.$, aromatic). ${ }^{13} \mathrm{C} \mathrm{NMR}\left(\mathrm{CDCl}_{3}, 75 \mathrm{MHz}\right) \delta$ $19.22,26.83,63.02,69.11,72.22,73.36,74.09,79.17,127.60,127.74,128.36$, $129.04,129.81,130.83,133.03,133.54,133.98,136.05,138.05$. ESI Q-TOF MS calcd for $\mathrm{C}_{32} \mathrm{H}_{38} \mathrm{KO}_{4} \mathrm{Si}[\mathrm{M}+\mathrm{K}]^{+} 553.218$, found 553.217. $[\alpha]_{\mathrm{D}}{ }^{24}-82.7^{\circ}$ (c 1.05, $\mathrm{CHCl}_{3}$ ). Anal. Calcd for $\mathrm{C}_{32} \mathrm{H}_{38} \mathrm{O}_{4} \mathrm{Si}$ : C, 74.67; H, 7.44. Found: C, 74.68; H, 7.67.

\section{$\beta$-Diol 19 and $\alpha$-Diol 20}

To a solution of allyl alcohol $17(2.20 \mathrm{~g}, 4.27 \mathrm{mmol})$ in dist. dichloromethane (21 $\mathrm{ml})$ were added $d i$-sodium hydrogenphosphate $(1.20 \mathrm{~g}, 8.55 \mathrm{mmol})$ and $80 \% \mathrm{~m}$ chloroperbenzoic acid $(1.03 \mathrm{~g}, 4.77 \mathrm{mmol})$ at $0{ }^{\circ} \mathrm{C}$. After stirring for $14 \mathrm{~h}$ at $\mathrm{rt}$ under nitrogen atmosphere, the reaction mixture was poured into saturated sodium sulfite aq. and then extracted with dichloromethane (x 3). The combined organic layer was washed with brine and dried over anhydrous sodium sulfate. Concentration of the solvent gave a crude oil, which was purified by silica gel column chromatography (ether / hexane $=30$ / 70) to afford a crude epoxy alcohol $\mathbf{1 8}(2.22 \mathrm{~g})$ as a colorless oil.

To a solution of the crude epoxy alcohol $18(2.22 \mathrm{~g})$ in dist. toluene $(21 \mathrm{ml})$ was added a solution of Red-Al ${ }^{\circledR}(65 \%$ in toluene, $2.5 \mathrm{ml}, 8.55 \mathrm{mmol})$ at $0{ }^{\circ} \mathrm{C}$. After stirring for $1.5 \mathrm{~h}$ at $0{ }^{\circ} \mathrm{C}$, the reaction mixture was added $10 \%$ acetic acid aq. at $0{ }^{\circ} \mathrm{C}$ and then poured into a mixture of saturated sodium hydrogen carbonate aq. and saturated potassium sodium tartrate aq. (1vol./1 vol.) at $0{ }^{\circ} \mathrm{C}$. After stirring for $1 \mathrm{~h} \mathrm{at} \mathrm{rt}$, the reaction mixture was extracted with ether (x 3). The combined organic layer was washed with water and dried over anhydrous sodium sulfate. Concentration of the solvent gave a crude oil, which was purified by silica gel column chromatography (ether / hexane $=30 / 70$ ) to afford allyl alcohol 17 (126 mg, 6\%) and a olefinic alcohol (1.92 g, 86\% in two steps) as a colorless oil.

To a solution of the olefinic alcohol $(3.30 \mathrm{~g}, 6.19 \mathrm{mmol})$ in ethanol $(62 \mathrm{ml})$ were added sodium hydrogen carbonate $(1.56 \mathrm{~g}, 18.6 \mathrm{mmol})$ and $10 \%$ palladium charcoal (620 $\mathrm{mg}$ ). After stirring for $4 \mathrm{~h}$ at $\mathrm{rt}$ under hydrogen atmosphere, the reaction mixture was passed through SuperCel ${ }^{\circledR}$. Concentration of the solvent gave a crude oil, which was purified by silica gel column chromatography (ether / hexane $=20 / 80$ ) to afford $\beta$-diol 19 and $\alpha$-diol 20 (total $3.31 \mathrm{~g}, 100 \%$ ) as a colorless oil. 
$\beta$-Diol 19. IR (KBr) $v_{\max } 3444,2929,2857,1968,1890,1831,1735,1455,1428$, 1363, 1111, 825, 742, 703, 612, $503 \mathrm{~cm}^{-1}$. 1H NMR $\left(\mathrm{CDCl}_{3}, 300 \mathrm{MHz}\right) \delta 1.04(9 \mathrm{H}$, $\left.\mathrm{s},{ }^{t} \mathrm{Bu}\right), 1.10-1.14(1 \mathrm{H}, \mathrm{m}, \mathrm{H}-4), 1.44-1.56(2 \mathrm{H}, \mathrm{m}, \mathrm{H}-3$ and $\mathrm{H}-4), 1.68-1.86(2 \mathrm{H}, \mathrm{m}$, H-2' and H-3), 1.92-2.15 (2H, br, -OH), 1.97 (1H, dddd, $J=14.5,8.0,8.0,5.0 \mathrm{~Hz}$, H-2'), 3.19 (1H, dd, $J=9.0,1.0 \mathrm{~Hz}, \mathrm{H}-1), 3.33$ (1H, dd, $J=10.0,4.0 \mathrm{~Hz}, \mathrm{H}-6), 3.39$ $(1 \mathrm{H}, \mathrm{dd}, J=10.0,6.5 \mathrm{~Hz}, \mathrm{H}-6), 3.54-3.63(1 \mathrm{H}, \mathrm{m}, \mathrm{H}-5), 3.72-3.82(1 \mathrm{H}, \mathrm{m}, \mathrm{H}-2)$, $3.743 .84\left(2 \mathrm{H}, \mathrm{m}, \mathrm{H}-3^{\prime}\right), 4.21\left(1 \mathrm{H}, \mathrm{dd}, J=8.0,4.0 \mathrm{~Hz}, \mathrm{H}-1^{\prime}\right), 4.48(1 \mathrm{H}, \mathrm{d}, J=12.0$ $\mathrm{Hz},-\mathrm{OCH} 2 \mathrm{Ph}), 4.53\left(1 \mathrm{H}, \mathrm{d}, J=12.0 \mathrm{~Hz},-\mathrm{OCH} \mathrm{H}_{2} \mathrm{Ph}\right), 7.23-7.74(15 \mathrm{H}, \mathrm{m}$, aromatic).

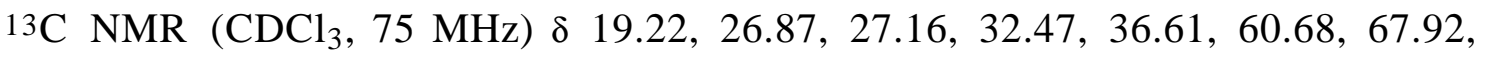
68.50, 72.69, 73.24, 76.57, 83.43, 127.53, 127.68, 127.77, 128.40, 129.71, 129.89, 133.39, 134.44, 135.89, 138.11. ESI Q-TOF MS calcd for $\mathrm{C}_{32} \mathrm{H}_{42} \mathrm{NaO}_{5} \mathrm{Si}[\mathrm{M}+\mathrm{Na}]^{+}$ 557.270, found 557.273. $[\alpha]_{\mathrm{D}}{ }^{25}-26.6^{\circ}$ (c 1.60, $\mathrm{CHCl}_{3}$ ). Anal. Calcd for $\mathrm{C}_{32} \mathrm{H}_{42} \mathrm{O}_{5} \mathrm{Si}$ : C, 71.87; H, 7.92. Found: C, 71.79; H, 7.86.

$\alpha$-Diol 20. IR (KBr) $v_{\max } 3424,3071,3032,2932,2893,2859,1968,1890,1831$, $1773,1467,1455,1428,1363,1317,1112,1084,824,742,703,613,505 \mathrm{~cm}^{-1} .{ }^{1} \mathrm{H}$ $\mathrm{NMR}\left(\mathrm{CDCl}_{3}, 400 \mathrm{MHz}\right) \delta 1.03\left(9 \mathrm{H}, \mathrm{s},{ }^{t} \mathrm{Bu}\right), 1.02-1.12(1 \mathrm{H}, \mathrm{m}, \mathrm{H}-4), 1.43-1.55(2 \mathrm{H}$, m, H-4 and H-2'), 1.67-1.75 (2H, m, H-3), 1.72-1.80 (1H, m, H-2'), 2.84 (1H, br-s, $-\mathrm{OH}), 3.09-3.27(1 \mathrm{H}, \mathrm{br}-\mathrm{m},-\mathrm{OH}), 3.32(1 \mathrm{H}, \mathrm{dd} J=10.0,4.0 \mathrm{~Hz}, \mathrm{H}-6), 3.38(1 \mathrm{H}, \mathrm{dd}$, $J=10.0,6.0 \mathrm{~Hz}, \mathrm{H}-6), 3.41(1 \mathrm{H}, \mathrm{dd}, J=9.0,4.5 \mathrm{~Hz}, \mathrm{H}-1), 3.48-3.58$ (2H, m, H-1' and H-5), 3.67-3.82 (2H, m, H-3'), 4.18 (1H, ddd, $J=9.0,4.5,4.0 \mathrm{~Hz}, \mathrm{H}-2), 4.46$ $(1 \mathrm{H}, \mathrm{d}, J=16.5 \mathrm{~Hz},-\mathrm{OCH} 2 \mathrm{Ph}), 4.51(1 \mathrm{H}, \mathrm{d}, J=16.5 \mathrm{~Hz},-\mathrm{OCH} 2 \mathrm{Ph}), 7.25-7.71$ $\left(15 \mathrm{H}, \mathrm{m}\right.$, aromatic). ${ }^{13 \mathrm{C} \mathrm{NMR}}\left(\mathrm{CDCl}_{3}, 100 \mathrm{MHz}\right) \delta 19.15,26.89,27.38,32.85$, $32.89,61.24,71.41,72.37,72.63,73.29,83.61,127.52,127.57,127.63,127.86$, $128.36,129.77,130.04,132.89,134.13,135.74,135.81,138.08$. ESI Q-TOF MS calcd for $\mathrm{C}_{32} \mathrm{H}_{42} \mathrm{NaO}_{5} \mathrm{Si}[\mathrm{M}+\mathrm{Na}]^{+} 557.270$, found 557.267. $[\alpha]_{\mathrm{D}}{ }^{25}-36.1^{\circ}(c 0.64$, $\mathrm{CHCl}_{3}$ ). Anal. Calcd for $\mathrm{C}_{32} \mathrm{H}_{42} \mathrm{O}_{5} \mathrm{Si}: \mathrm{C}, 71.87 ; \mathrm{H}, 7.92$. Found: C, 71.79; H, 7.86.

\section{Alcohol 21}

To a solution of $\beta$-diol $19(380 \mathrm{mg}, 0.71 \mathrm{mmol})$ in dichloromethane $(3.5 \mathrm{ml})$ were added benzaldehyde dimethylacetal $(0.21 \mathrm{ml}, 1.42 \mathrm{mmol})$ and 10-comphorsulfonic acid (33 mg, $0.14 \mathrm{mmol}$ ) at $\mathrm{rt}$. After stirring for $14 \mathrm{~h}$, the reaction mixture was poured into saturated sodium hydrogen carbonate aq. at $0{ }^{\circ} \mathrm{C}$ and then extracted with dichloromethane (x 3). The combined organic layer was washed with brine and dried over anhydrous sodium sulfate. Concentration of the solvent gave a crude oil, which was purified by 
silica gel column chromatography (ether / hexane $=10 / 90$ ) to afford a benzylidene acetal (438 mg, 99\%) as a colorless oil.

To the benzylidene acetal $(590 \mathrm{mg}, 0.95 \mathrm{mmol})$ in round flask was added dropwise a solution of $1.0 \mathrm{M} \mathrm{BH}_{3}$. THF in THF $(9.5 \mathrm{ml}, 9.47 \mathrm{mmol})$ at $\mathrm{rt}$. After refluxing for $7 \mathrm{~h}$, the reaction mixture was added methanol at $0{ }^{\circ} \mathrm{C}$ much carefully. Concentration of the solvent gave a crude oil, which was purified by silica gel column chromatography (ether / hexane $=15 / 85)$ to afford alcohol $21(525 \mathrm{mg}, 89 \%)$ as a colorless oil.

Alcohol 21. IR (KBr) $v_{\max } 3449,3069,3032,2932,2858,1961,1890,1825,1734$, 1455, 1428, 1363, 1111, 1029, 825, 740, 703, 612, $503 \mathrm{~cm}^{-1}$. 1H NMR $\left(\mathrm{CDCl}_{3}, 300\right.$ MHz) $\delta 1.02\left(9 \mathrm{H}, \mathrm{s},{ }^{t} \mathrm{Bu}\right), 1.08-1.22(1 \mathrm{H}, \mathrm{m}, \mathrm{H}-4), 1.36-1.52(2 \mathrm{H}, \mathrm{m}, \mathrm{H}-3$ and $\mathrm{H}-4)$, 1.76-1.96 (2H, m, H-3 and H-2'), 2.04-2.16 (1H, m, H-2'), $3.05(1 \mathrm{H}, \mathrm{br},-\mathrm{OH}), 3.30$ $(1 \mathrm{H}, \mathrm{dd}, J=9.5,3.5 \mathrm{~Hz}, \mathrm{H}-6), 3.34(1 \mathrm{H}, \mathrm{dd}, J=9.0,1.0 \mathrm{~Hz}, \mathrm{H}-1), 3.44(1 \mathrm{H}, \mathrm{dd}, J=$ 9.5, 7.0 Hz, H-6), 3.47-3.55 (1H, m, H-2), 3.59-3.69 (1H, m, H-5), 3.85 (1H, ddd, $J$ $=10.5,7.5,3.5 \mathrm{~Hz}, \mathrm{H}-3$ ') $4.02(1 \mathrm{H}, \mathrm{ddd}, J=10.5,9.0,4.5 \mathrm{~Hz}, \mathrm{H}-3$ ') $4.23(1 \mathrm{H}$, ddd, $\left.J=6.0,4.5,1.0 \mathrm{~Hz}, \mathrm{H}-1^{\prime}\right), 4.37\left(1 \mathrm{H}, \mathrm{d}, J=11.5 \mathrm{~Hz},-\mathrm{OCH}_{2} \mathrm{Ph}\right), 4.45(1 \mathrm{H}, \mathrm{d}, J$ $\left.=12.0 \mathrm{~Hz},-\mathrm{OCH}{ }_{2} \mathrm{Ph}\right), 4.50\left(1 \mathrm{H}, \mathrm{d}, J=11.5 \mathrm{~Hz},-\mathrm{OCH}_{2} \mathrm{Ph}\right), 4.56(1 \mathrm{H}, \mathrm{d}, J=12.0$ $\left.\mathrm{Hz},-\mathrm{OCH} \mathrm{H}_{2} \mathrm{Ph}\right), 7.18-7.70\left(20 \mathrm{H}, \mathrm{m}\right.$, aromatic). ${ }^{13} \mathrm{C} \mathrm{NMR}\left(\mathrm{CDCl}_{3}, 75 \mathrm{MHz}\right) \delta 19.15$, $26.93,27.00,32.86,33.85,58.46,68.36,71.82,72.59,73.21,74.81,77.14,84.24$, $127.33,127.49,127.57,127.68,127.77,128.21,128.36,129.64,129.74,133.82$, 134.76, 135.82, 135.89, 138.18, 139.02. ESI Q-TOF MS calcd for $\mathrm{C}_{39} \mathrm{H}_{49} \mathrm{O}_{5} \mathrm{Si}$ $[\mathrm{M}+\mathrm{H}]^{+}$625.335, found 625.370. $[\alpha]_{\mathrm{D}}^{24}-11.9^{\circ}\left(c\right.$ 1.13, $\left.\mathrm{CHCl}_{3}\right)$. Anal. Calcd for $\mathrm{C}_{39} \mathrm{H}_{48} \mathrm{O}_{5} \mathrm{Si}: \mathrm{C}, 74.96 ; \mathrm{H}, 7.74$. Found: C, 74.96; H, 7.87.

\section{Aldehyde 22}

To a solution of 21 (623 mg, $1.00 \mathrm{mmol})$ in DMSO (5 ml) was added IBX (419 $\mathrm{mg}, 1.50 \mathrm{mmol}$ ). After stirring for $5 \mathrm{~h}$ at $\mathrm{rt}$, the reaction mixture was diluted with $\mathrm{H}_{2} \mathrm{O}$ $(10 \mathrm{ml})$. The resulting emulsion was passed through SuperCel ${ }^{\circledR}$ and washed with ether. The filtrate was extracted with ether (x 3). Concentration of the solvent gave a crude oil, which was purified by silica gel column chromatography (ether / hexane $=20 / 80$ ) to afford aldehyde 22 (569 $\mathrm{mg}, 92 \%$ ) as a colorless oil.

Aldehyde 22. IR (KBr) $v_{\max } 3069,3032,2932,2893,2858,1720,1473,1455,1428$, 1363, 1111, 1028, 825, 741, 703, 612, $509 \mathrm{~cm}^{-1}$. $1 \mathrm{H} \mathrm{NMR}\left(\mathrm{CDCl}_{3}, 300 \mathrm{MHz}\right) \delta 1.05$ $\left(9 \mathrm{H}, \mathrm{s},{ }^{t} \mathrm{Bu}\right), 1.10-1.26(1 \mathrm{H}, \mathrm{m}, \mathrm{H}-4), 1.35-1.47(1 \mathrm{H}, \mathrm{m}, \mathrm{H}-3), 1.48-1.57(1 \mathrm{H}, \mathrm{m}, \mathrm{H}-$ 4), 1.77-1.87 (1H, m, H-3), 2.67 (1H, ddd, $J=16.5,4.5,3.5 \mathrm{~Hz}, \mathrm{H}-2$ '), $2.83(1 \mathrm{H}$, ddd, $\left.J=16.5,6.0,1.5 \mathrm{~Hz}, \mathrm{H}-2^{\prime}\right), 3.29(1 \mathrm{H}, \mathrm{dd}, J=9.0,2.0 \mathrm{~Hz}, \mathrm{H}-1), 3.28(1 \mathrm{H}, \mathrm{m}$, 
H-6), 3.40-3.51 (2H, m, H-5 and H-6), 4.03 (1H, ddd, $J=10.5,9.0,4.5 \mathrm{~Hz}, \mathrm{H}-2)$, $4.41(1 \mathrm{H}, \mathrm{d}, J=11.5 \mathrm{~Hz},-\mathrm{OCH} 2 \mathrm{Ph}), 4.45(1 \mathrm{H}, \mathrm{d}, J=12.0 \mathrm{~Hz},-\mathrm{OCH} 2 \mathrm{Ph}), 4.51(1 \mathrm{H}$, $\left.\mathrm{d}, J=12.0 \mathrm{~Hz},-\mathrm{OCH}_{2} \mathrm{Ph}\right), 4.52\left(1 \mathrm{H}, \mathrm{d}, J=11.5 \mathrm{~Hz},-\mathrm{OCH}_{2} \mathrm{Ph}\right), 4.53(1 \mathrm{H}, \mathrm{ddd}, J=$ 6.0, 4.5, $\left.2.0 \mathrm{~Hz}, \mathrm{H}-1^{\prime}\right), 7.15-7.71(20 \mathrm{H}, \mathrm{m}$, aromatic), $9.85(1 \mathrm{H}, \mathrm{dd}, J=2.5,1.5 \mathrm{~Hz}$, $\mathrm{H}-3$ '). ${ }^{13} \mathrm{C} \mathrm{NMR}\left(\mathrm{CDCl}_{3}, 75 \mathrm{MHz}\right) \delta 19.09,26.90,27.45,32.82,45.00,68.12$, 72.18, 72.64, 73.06, 73.24, 77.11, 84.20, 127.47, 127.53, 127.61, 127.63, 127.67, $128.24,128.32,129.64,129.73,133.76,134.55,135.78,135.87,138.32,138.44$, 201.80. ESI Q-TOF MS calcd for $\mathrm{C}_{39} \mathrm{H}_{46} \mathrm{NaO}_{5} \mathrm{Si}[\mathrm{M}+\mathrm{Na}]^{+}$645.301, found 645.353. $[\alpha]_{\mathrm{D}}{ }^{24}-13.3^{\circ}\left(c\right.$ 1.30, $\left.\mathrm{CHCl}_{3}\right)$. Anal. Calcd for $\mathrm{C}_{39} \mathrm{H}_{46} \mathrm{O}_{5} \mathrm{Si}: \mathrm{C}, 75.20 ; \mathrm{H}, 7.44$. Found: C, 75.07; H, 7.66.

\section{E'FH' ring 25}

To a solution of E' ring-aldehyde $12(603 \mathrm{mg}, 2.53 \mathrm{mmol})$, which was treatment with toluene azeotrope in advance, in dist. tetrahydrofuran $(25 \mathrm{ml})$ was added dropwise a solution of $1.59 \mathrm{M} n \mathrm{BuLi}$ in Hexane $(1.59 \mathrm{ml}, 2.53 \mathrm{mmol})$ at $-78{ }^{\circ} \mathrm{C}$ under nitrogen atmosphere. After stirring for $30 \mathrm{~min}$ at $-78{ }^{\circ} \mathrm{C}$, a solution of aldehyde $22(1.05 \mathrm{~g}, 1.69$ $\mathrm{mmol}$ ), which was treatment with toluene azeotrope in advance, in dist. tetrahydrofuran $\left(7 \mathrm{ml}\right.$ ) was added to the reaction mixture over $10 \mathrm{~min}$. After stirring for $35 \mathrm{~min}$ at $-78{ }^{\circ} \mathrm{C}$, the reaction mixture was poured into saturated sodium hydrogen carbonate aq. at $0{ }^{\circ} \mathrm{C}$ and then extracted with ether (x 3). The combined organic layer was washed with brine and dried over anhydrous sodium sulfate. Concentration of the solvent gave a crude oil, which was purified by silica gel column chromatography (ethyl acetate / hexane $=5$ / 95 to $20 / 80)$ to afford an propargyl alcohol $(1.24 \mathrm{~g}, 86 \%)$ as a colorless oil, recovered $\mathrm{E}^{\prime}$ ring-enyne 12 (258 mg, 43\%) and recovered H' ring-aldehyde 22 (151 mg, 14\%).

To a solution of the propargyl alcohol $(250 \mathrm{mg}, 0.29 \mathrm{mmol})$ in tetrahydrofuran $(2.9$ $\mathrm{ml})$ was added $1.0 \mathrm{M}$ tetrabutylammonium fluoride in tetrahydrofuran $(0.58 \mathrm{ml}, 0.58$ $\mathrm{mmol}$ ) at $0{ }^{\circ} \mathrm{C}$. After stirring for $7 \mathrm{~h}$ at $\mathrm{rt}$ under nitrogen atmosphere, the reaction mixture was poured into saturated ammonium chloride aq. at $0{ }^{\circ} \mathrm{C}$ and then extracted with ether $(\mathrm{x}$ $3)$. The combined organic layer was washed with brine and dried over anhydrous sodium sulfate. Concentration of the solvent gave a crude diol, which was used in next step without further purification.

To a solution of the crude diol in dichloromethane $(4 \mathrm{ml})$ were added pyridine $(0.5$ $\mathrm{ml})$, acetic anhydride $(0.2 \mathrm{ml})$ and $N, N$-dimethylaminopyridine $(10 \mathrm{mg})$. After stirring for $2 \mathrm{~h}$ at $\mathrm{rt}$, the reaction mixture was poured into saturated sodium hydrogen carbonate aq. at $0{ }^{\circ} \mathrm{C}$ and then extracted with dichloromethane (x 3). The combined organic layer was washed with brine and dried over anhydrous sodium sulfate. Concentration of the 
solvent gave a crude oil, which was purified by silica gel column chromatography (ethyl acetate / hexane $=30 / 70)$ to afford diacetate $\mathbf{2 3}(201 \mathrm{mg}, 98 \%)$ as a colorless oil.

To a solution of diacetate 23 (37 mg, $0.052 \mathrm{mmol})$ in methanol (2 ml) was added pyridinium $p$-toluenesulfonate $(13 \mathrm{mg}, 0.052 \mathrm{mmol})$. After stirring for $30 \mathrm{~min}$ at $\mathrm{rt}$, the reaction mixture was poured into saturated sodium hydrogen carbonate aq. at $0{ }^{\circ} \mathrm{C}$ and then extracted with ether (x 3). The combined organic layer was washed with brine and dried over anhydrous sodium sulfate. Concentration of the solvent gave a crude oil, which was purified by short silica gel column chromatography to afford a crude alcohol.

To a solution of the crude alcohol in dry dichloromethane $(2 \mathrm{ml})$ was added a solution of $\mathrm{Co}_{2}(\mathrm{CO})_{8}(36 \mathrm{mg}, 0.10 \mathrm{mmol})$ in dry dichloromethane $(1 \mathrm{ml})$ at $0{ }^{\circ} \mathrm{C}$ under nitrogen atmosphere. After stirring for $1.5 \mathrm{~h}$ at $\mathrm{rt}$, the solvent was concentrated. The residue was purified by silica gel column chromatography (ether / hexane $=40 / 60$ ) to afford acetylene cobalt complex 24 (46 mg, 96\% in two steps) as a reddish brown oil.

To a solution of acetylene cobalt complex $24(71 \mathrm{mg}, 0.077 \mathrm{mmol})$ in dry dichloromethane $(7.7 \mathrm{ml})$ was added a solution of $\mathrm{BF}_{3} \cdot \mathrm{OEt}_{2}(0.20 \mathrm{M}$ in $1,2-$ dichloroethane, $0.39 \mathrm{ml}, 0.077 \mathrm{mmol}$ ) at $0{ }^{\circ} \mathrm{C}$ under nitrogen atmosphere. After stirring for $10 \mathrm{~min}$ at $0{ }^{\circ} \mathrm{C}$ and for $30 \mathrm{~min}$ at $\mathrm{rt}$, the reaction mixture was poured into saturated sodium hydrogen carbonate aq. at $0{ }^{\circ} \mathrm{C}$ and then extracted with dichloromethane (x 3). The combined organic layer was washed with brine and dried over anhydrous sodium sulfate. Concentration of the solvent gave a crude oil, which was purified by silica gel column chromatography (ether / hexane = 30 / 70) to afford E'FH' ring 25 (51 mg, 77\%) as a reddish brown oil.

E'FH' ring 25. IR (KBr) $v_{\max }$ 2931, 2855, 2361, 2090, 2051, 2026, 1741, 1560, $1456,1373,1239,1095,736,698,661,518,498 \mathrm{~cm}^{-1} .{ }^{1} \mathrm{H} \mathrm{NMR}\left(\mathrm{CDCl}_{3}, 400 \mathrm{MHz}\right) \delta$ 1.27-1.38 (1H, m, H-3), 1.42-1.60 (2H, m, H-2), 1.42-1.60 (2H, m, H-16 and H-17), 1.75-1.86 (1H, m, H-3 and H-17), $1.92\left(3 \mathrm{H}, \mathrm{s},-\mathrm{OCOCH}_{3}\right), 1.97(1 \mathrm{H}$, ddd, $J=14.5$, 10.5, $3.5 \mathrm{~Hz}, \mathrm{H}-12), 2.26-2.38$ (3H, m, H-6, H-12 and H-16), 2.69 (1H, br-dd, $J=$ 13.5, $9.5 \mathrm{~Hz}, \mathrm{H}-6), 3.21(1 \mathrm{H}, \mathrm{dd}, J=10.0,4.5 \mathrm{~Hz}, \mathrm{H}-4), 3.28(1 \mathrm{H}, \mathrm{dd}, J=10.0,4.0$ Hz, H-5), 3.25-3.35 (1H, m, H-1), 3.40-3.47 (1H, m, H-6), 3.53-3.62 (3H, m, H-14, $\mathrm{H}-18$ and $\mathrm{H}-19), 3.81-3.91(2 \mathrm{H}, \mathrm{m}, \mathrm{H}-1$ and $\mathrm{H}-13), 4.54\left(2 \mathrm{H}, \mathrm{s},-\mathrm{OCH} \mathrm{H}_{2} \mathrm{Ph}\right), 4.59(1 \mathrm{H}$, $\left.\mathrm{d}, J=12.0 \mathrm{~Hz},-\mathrm{OCH}_{2} \mathrm{Ph}\right), 4.67(1 \mathrm{H}, \mathrm{d}, J=12.0 \mathrm{~Hz},-\mathrm{OCH} 2 \mathrm{Ph}), 4.79(1 \mathrm{H}, \mathrm{dd}, J=$ $11.5,1.5 \mathrm{~Hz}, \mathrm{H}-11), 4.91(1 \mathrm{H}, \mathrm{ddd}, J=10.0,9.5,5.0 \mathrm{~Hz}, \mathrm{H}-15), 5.86$ (1H, ddd, $J=$ 11.0, 9.5, $7.5 \mathrm{~Hz}, \mathrm{H}-7), 6.76(1 \mathrm{H}, \mathrm{d}, J=11.0 \mathrm{~Hz}, \mathrm{H}-8), 7.22-7.34(10 \mathrm{H}, \mathrm{m}$, aromatic). ${ }^{13} \mathrm{C} \mathrm{NMR}\left(\mathrm{CDCl}_{3}, 100 \mathrm{MHz}\right) \delta 21.16,25.56,27.56,29.19,29.28,29.87$, $38.70,67.92,68.83,71.72,72.79,73.48,74.03,74.64,77.50,78.13,80.06,80.61$, $86.78,99.72,127.55,127.62,127.70,128.34,130.51,130.70,138.40,138.64$, 169.79, 199.41. ESI Q-TOF MS calcd for $\mathrm{C}_{41} \mathrm{H}_{43} \mathrm{Co}_{2} \mathrm{O}_{13}[\mathrm{M}+\mathrm{H}]^{+} 861.137$, found

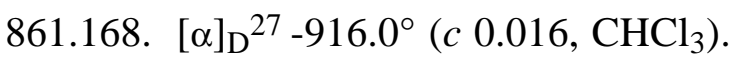




\section{Ketone 26, Conjugated Enone 27 and Diene 28}

To a PORTABLE REACTOR (TVS-N2 type, TAIATSU TECHNO ${ }^{\circledR}$ CORPORATION, Japan) was placed a solution of acetylene cobalt complex $25(29 \mathrm{mg}, 0.034 \mathrm{mmol})$ in benzene $(17 \mathrm{ml})$. After stirring for $4.5 \mathrm{~h}$ at $65{ }^{\circ} \mathrm{C}$ under $100 \mathrm{~kg} / \mathrm{cm}^{2}$ hydrogen atmosphere, the pressure was reduced to ambient pressure at $\mathrm{rt}$. The reaction mixture was passed through SuperCel ${ }^{\circledR}$ and concentrared to give a crude oil, which was purified by preparative thin layer chromatography to afford SM (2.1 mg, 7\%), diene 27 (2.9 $\mathrm{mg}$, $15 \%)$, ketone 26 (7.3 $\mathrm{mg}, 37 \%)$ and conjugated enone 28 (0.7 $\mathrm{mg}, 4 \%)$.

Ketone 26. IR (KBr) $v_{\max } 2931,2858,1738,1716,1455,1372,1240,1092,1038$, $738,699 \mathrm{~cm}^{-1} .{ }^{1} \mathrm{H} \mathrm{NMR}\left(\mathrm{CDCl}_{3}, 400 \mathrm{MHz}\right) \delta 1.27(1 \mathrm{H}, \mathrm{dddd}, J=12.0,12.0,11.0$, $5.0 \mathrm{~Hz}, \mathrm{H}-3), 1.40-1.50(2 \mathrm{H}, \mathrm{m}, \mathrm{H}-16$ and 17$), 1.47-1.53(2 \mathrm{H}, \mathrm{m}, \mathrm{H}-2), 1.77(1 \mathrm{H}$, $\mathrm{dm}, J=8.5 \mathrm{~Hz}, \mathrm{H}-17), 1.87\left(3 \mathrm{H}, \mathrm{s},-\mathrm{OCOCH}_{3}\right), 1.91-1.96(2 \mathrm{H}, \mathrm{m}, \mathrm{H}-12), 1.97-2.03$ $(1 \mathrm{H}, \mathrm{m}, \mathrm{H}-3), 2.20(1 \mathrm{H}, \mathrm{ddd}, J=13.5,6.5,3.0 \mathrm{~Hz}, \mathrm{H}-6), 2.29(1 \mathrm{H}$, ddd, $J=8.0$, 5.0, 3.0 Hz, H-16), 2.56 (1H, dd, $J=10.5,7.0 \mathrm{~Hz}, \mathrm{H}-9), 2.97$ (1H, ddd, $J=13.5$, 10.5, $3.5 \mathrm{~Hz}, \mathrm{H}-6), 2.97(1 \mathrm{H}, \mathrm{ddd}, J=11.0,9.5,4.0 \mathrm{~Hz}, \mathrm{H}-4), 3.27(1 \mathrm{H}, \mathrm{ddd}, J=$ 9.5, 3.5, 3.0 Hz, H-5), $3.28(1 \mathrm{H}, \mathrm{ddd}, J=11.5,11.5,3.0 \mathrm{~Hz}, \mathrm{H}-1), 3.43(1 \mathrm{H}, \mathrm{dd}, J=$ 12.5, $7.5 \mathrm{~Hz}, \mathrm{H}-19), 3.50$ (1H, dd, $J=9.5,2.5 \mathrm{~Hz}, \mathrm{H}-14), 3.55-3.60$ (1H, m, H-18), $3.57(1 \mathrm{H}, \mathrm{dd}, J=12.5,5.5 \mathrm{~Hz}, \mathrm{H}-19), 3.73(1 \mathrm{H}, \mathrm{ddd}, J=7.0,5.5,2.5 \mathrm{~Hz}, \mathrm{H}-13)$, $3.83(1 \mathrm{H}, \mathrm{dm}, J=11.5 \mathrm{~Hz}, \mathrm{H}-1), 3.87(1 \mathrm{H}, \mathrm{dd}, J=8.5,5.5 \mathrm{~Hz}, \mathrm{H}-11), 3.97(1 \mathrm{H}, \mathrm{dd}$, $J=10.5,10.5 \mathrm{~Hz}, \mathrm{H}-9), 4.51\left(2 \mathrm{H}, \mathrm{s},-\mathrm{OCH}_{2} \mathrm{Ph}\right), 4.53(1 \mathrm{H}, \mathrm{d}, J=12.0 \mathrm{~Hz}$, $-\mathrm{OCH} 2 \mathrm{Ph}), 4.58\left(1 \mathrm{H}, \mathrm{d}, J=12.0 \mathrm{~Hz},-\mathrm{OCH}_{2} \mathrm{Ph}\right), 4.86(1 \mathrm{H}, \mathrm{ddd}, J=10.0,9.5,5.0$ $\mathrm{Hz}, \mathrm{H}-15), 5.62(1 \mathrm{H}, \mathrm{ddd}, J=10.5,10.5,7.0 \mathrm{~Hz}, \mathrm{H}-8), 5.71(1 \mathrm{H}$, ddd, $J=10.5$, $10.5,6.5 \mathrm{~Hz}, \mathrm{H}-7), 7.25-7.37\left(10 \mathrm{H}, \mathrm{m}\right.$, aromatic). ${ }^{13} \mathrm{C} \mathrm{NMR}\left(\mathrm{CDCl}_{3}, 100 \mathrm{MHz}\right) \delta$ $21.13,25.51,27.36,28.67,29.11,29.40,32.76,36.90,68.15,68.27,71.41,72.87$, $73.43,73.54,75.20,77.60,78.83,79.66,82.16,124.57,127.63,127.99,128.32$, 128.38, 128.69, 138.12, 138.30, 169.80, 211.48. ESI Q-TOF MS calcd for $\mathrm{C}_{35} \mathrm{H}_{44} \mathrm{NaO}_{8}[\mathrm{M}+\mathrm{Na}]^{+} 615.293$, found 615.284. $[\alpha]_{\mathrm{D}}{ }^{24}+118.7^{\circ}\left(c 0.30, \mathrm{CHCl}_{3}\right)$.

Diene 28. IR (KBr) $v_{\max } 2926,2854,1739,1455,1373,1240,1145,1090,1046$, 738, $698 \mathrm{~cm}^{-1} .{ }^{1} \mathrm{H} \mathrm{NMR}\left(\mathrm{CDCl}_{3}, 400 \mathrm{MHz}\right) \delta 1.30-1.46(3 \mathrm{H}, \mathrm{m}, \mathrm{H}-3, \mathrm{H}-16$ and 17), 1.45-1.55 (2H, m, H-2), $1.78(1 \mathrm{H}, \mathrm{dm}, J=7.5 \mathrm{~Hz}, \mathrm{H}-17), 1.89\left(3 \mathrm{H}, \mathrm{s},-\mathrm{OCOCH}_{3}\right)$, 1.90-1.95 (2H, m, H-12), 1.94-1.99 (1H, m, H-3), 2.22 (1H, br-ddd, $J=13.5,6.0$, $4.0 \mathrm{~Hz}, \mathrm{H}-6), 2.30$ (1H, ddd, $J=8.5,5.0,3.0 \mathrm{~Hz}, \mathrm{H}-16), 2.74(1 \mathrm{H}$, ddd, $J=13.5$, 10.5, $3.5 \mathrm{~Hz}, \mathrm{H}-6), 3.07$ (1H, ddd, $J=9.5,4.0,3.5 \mathrm{~Hz}, \mathrm{H}-5), 3.20(1 \mathrm{H}, \mathrm{ddd}, J=$ $10.5,9.5,4.0 \mathrm{~Hz}, \mathrm{H}-4), 3.25$ (1H, ddd, $J=11.5,11.5,3.0 \mathrm{~Hz}, \mathrm{H}-1), 3.39$ (1H, dd, $J$ 
$=10.0,2.5 \mathrm{~Hz}, \mathrm{H}-14), 3.43(1 \mathrm{H}, \mathrm{dd}, J=12.0,7.0 \mathrm{~Hz}, \mathrm{H}-19), 3.53-3.58(1 \mathrm{H}, \mathrm{m}, \mathrm{H}-$ 18), 3.57 (1H, dd, $J=12.0,5.5 \mathrm{~Hz}, \mathrm{H}-19), 3.78$ (1H, td, $J=6.0,2.5 \mathrm{~Hz}, \mathrm{H}-13), 3.83$ $(1 \mathrm{H}, \mathrm{dm}, J=11.5 \mathrm{~Hz}, \mathrm{H}-1), 3.96(1 \mathrm{H}, \mathrm{dt}, J=7.0,7.0 \mathrm{~Hz}, \mathrm{H}-11), 4.53(1 \mathrm{H}, \mathrm{d}, J=$ $\left.12.0 \mathrm{~Hz},-\mathrm{OCH} \mathrm{H}_{2} \mathrm{Ph}\right), 4.54\left(2 \mathrm{H}, \mathrm{s},-\mathrm{OCH} \mathrm{H}_{2} \mathrm{Ph}\right), 4.58\left(1 \mathrm{H}, \mathrm{d}, J=12.0 \mathrm{~Hz},-\mathrm{OCH} \mathrm{H}_{2} \mathrm{Ph}\right)$, $4.90(1 \mathrm{H}, \mathrm{ddd}, J=10.0,10.0,5.0 \mathrm{~Hz}, \mathrm{H}-15), 5.58(1 \mathrm{H}, \mathrm{dd}, J=11.0,7.0 \mathrm{~Hz}, \mathrm{H}-10)$, $5.79(1 \mathrm{H}, \mathrm{ddd}, J=11.0,10.5,6.0 \mathrm{~Hz}, \mathrm{H}-7), 5.97(1 \mathrm{H}, \mathrm{br}-\mathrm{d}, J=11.0 \mathrm{~Hz}, \mathrm{H}-9), 6.01$ $(1 \mathrm{H}$, br-d, $J=11.0 \mathrm{~Hz}, \mathrm{H}-8), 7.25-7.39$ (10H, m, aromatic). ${ }^{13} \mathrm{C} \mathrm{NMR}\left(\mathrm{CDCl}_{3}, 100\right.$ MHz) $\delta 21.13,26.10,27.47,29.07,31.65,33.23$, 38.17, 67.90, 68.50, 71.15, 72.90, $73.40,77.56,78.83,80.54,80.74,80.90,127.59,127.63,127.94,128.35,129.46$, $129.78,130.27,134.74,138.35,138.51,169.80$. ESI Q-TOF MS calcd for $\mathrm{C}_{35} \mathrm{H}_{44} \mathrm{NaO}_{7}[\mathrm{M}+\mathrm{Na}]^{+} 599.298$, found 599.302. $[\alpha]_{\mathrm{D}}{ }^{25}-74.6^{\circ}\left(c 0.25, \mathrm{CHCl}_{3}\right)$. Anal. Calcd for $\mathrm{C}_{35} \mathrm{H}_{44} \mathrm{O}_{7}$ : C, 72.89; H, 7.69. Found: C, 72.88; H, 7.72.

Conjugated Enone 27. IR (KBr) $v_{\max } 2928,2855,1737,1653,1455,1373,1241$, 1093, 1040, 738, $699 \mathrm{~cm}^{-1}$. ${ }^{1} \mathrm{H}$ NMR $\left(\mathrm{CDCl}_{3}, 400 \mathrm{MHz}\right) \delta 1.29-1.40$ (1H, m, H-3), 1.40-1.50 (2H, m, H-16 and 17), 1.46-1.58 (2H, m, H-2), 1.74-1.78 (1H, m, H-17), 1.80-1.94 (1H, m, H-3), 1.80-1.94 (2H, m, H-12), 1.88 (3H, s, - $\left.\mathrm{OCOCH}_{3}\right), 2.25-2.31$ (1H, m, H-16), 2.70 (1H, ddd, $J=13.5,9.5,7.0 \mathrm{~Hz}, \mathrm{H}-6), 2.79(1 \mathrm{H}, \mathrm{dd}, J=13.5$, $6.0 \mathrm{~Hz}, \mathrm{H}-10), 2.85-2.93$ (1H, m, H-10), 2.90 (1H, br-ddd, $J=13.5,9.5,2.0 \mathrm{~Hz}, \mathrm{H}-$ 6), 3.07 (1H, ddd, $J=10.5,9.5,4.5 \mathrm{~Hz}, \mathrm{H}-4), 3.23(1 \mathrm{H}$, ddd, $J=9.5,7.0,2.0 \mathrm{~Hz}$, H-5), 3.28 (1H, ddd, $J=11.5,11.5,3.0 \mathrm{~Hz}, \mathrm{H}-1), 3.43(1 \mathrm{H}, \mathrm{dd}, J=12.0,3.5 \mathrm{~Hz}, \mathrm{H}-$ 19), 3.44 (1H, dd, $J=9.5,2.5 \mathrm{~Hz}, \mathrm{H}-14), 3.52-3.58(1 \mathrm{H}, \mathrm{m}, \mathrm{H}-18), 3.56$ (1H, dd, $J=$ 12.0, $5.5 \mathrm{~Hz}, \mathrm{H}-19), 3.75$ (1H, ddd, $J=7.5,4.5,2.5 \mathrm{~Hz}, \mathrm{H}-13), 3.83(1 \mathrm{H}, \mathrm{dm}, J=$ $11.5 \mathrm{~Hz}, \mathrm{H}-1), 3.94(1 \mathrm{H}, \mathrm{ddt}, J=7.5,4.5,2.5 \mathrm{~Hz}, \mathrm{H}-11), 4.53(1 \mathrm{H}, \mathrm{d}, J=11.5 \mathrm{~Hz}$, $\left.-\mathrm{OCH}{ }_{2} \mathrm{Ph}\right), 4.53\left(1 \mathrm{H}, J=12.0 \mathrm{~Hz},-\mathrm{OCH}_{2} \mathrm{Ph}\right), 4.56\left(1 \mathrm{H}, \mathrm{d}, J=11.5 \mathrm{~Hz},-\mathrm{OCH}{ }_{2} \mathrm{Ph}\right)$, $4.57\left(1 \mathrm{H}, \mathrm{d}, J=12.0 \mathrm{~Hz},-\mathrm{OCH}{ }_{2} \mathrm{Ph}\right), 4.85(1 \mathrm{H}, \mathrm{ddd}, J=10.0,9.5,5.0 \mathrm{~Hz}, \mathrm{H}-15)$, $6.11(1 \mathrm{H}, \mathrm{d}, J=12.0 \mathrm{~Hz}, \mathrm{H}-8), 6.32(1 \mathrm{H}, \mathrm{dt}, J=12.0,9.5 \mathrm{~Hz}, \mathrm{H}-7), 7.26-7.38(10 \mathrm{H}$, $\mathrm{m}$, aromatic). ${ }^{13} \mathrm{C} \mathrm{NMR}\left(\mathrm{CDCl}_{3}, 100 \mathrm{MHz}\right) \delta 21.14,25.58,27.39,29.10,29.68$, $30.67,32.43,37.18,49.98,67.99,68,45,71.91,72.86,73.43,74.21,76.25,77.45$, $80.25,80.35,127.59,127.68,127.93,128.34,128.37,134.71,138.33,138.40$, 139.74, 169.84, 202.29. ESI Q-TOF MS calcd for $\mathrm{C}_{35} \mathrm{H}_{44} \mathrm{NaO}_{8}[\mathrm{M}+\mathrm{Na}]^{+}$615.293, found $615.290 .[\alpha]_{\mathrm{D}}^{26}+16.8^{\circ}\left(c 0.12, \mathrm{CHCl}_{3}\right)$.

\section{E'FGH' ring 5}

To a solution of ketone $26(7.9 \mathrm{mg}, 0.013 \mathrm{mmol})$ in methanol $(1 \mathrm{ml})$ was added $\mathrm{K}_{2} \mathrm{CO}_{3}$ $(3 \mathrm{mg}, 0.022 \mathrm{mmol})$. After stirring for $3 \mathrm{~h}$ at $\mathrm{rt}$, the reaction mixture was poured into saturated ammonium chloride aq. at $0{ }^{\circ} \mathrm{C}$ and then extracted with ether (x 3). The 
combined organic layer was washed with brine and dried over anhydrous sodium sulfate. Concentration of the solvent gave a crude oil, which was purified by preparative thin layer chromatography to afford a hydroxyketone $(7.2 \mathrm{mg}, 99 \%)$.

To a solution of the hydroxyketone $(7.2 \mathrm{mg}, 0.013 \mathrm{mmol})$ and triethylsilane $(0.13 \mathrm{ml}$, $0.81 \mathrm{mmol})$ in dry acetonitrile $(1.3 \mathrm{ml})$ was added $\mathrm{BF}_{3} \cdot \mathrm{OEt}_{2}(0.79 \mathrm{M}$ in $1,2-$ dichloroethane, $0.1 \mathrm{ml}, 0.079 \mathrm{mmol}$ ) at $-15{ }^{\circ} \mathrm{C}$ under nitrogen atmosphere. After stirring for $20 \mathrm{~min}$ at $-15^{\circ} \mathrm{C}$ and for $30 \mathrm{~min}$ at $\mathrm{rt}$, the reaction mixture was poured into saturated sodium hydrogen carbonate aq. at $0{ }^{\circ} \mathrm{C}$ and then extracted with ether (x 3). The combined organic layer was washed with brine and dried over anhydrous sodium sulfate. Concentration of the solvent gave a crude oil, which was purified by preparative thin layer chromatography to afford E'FGH' ring 5 (4.0 mg, 57\%) as a white solid.

E'FGH' ring 5. Mp 113.0-113.5 ${ }^{\circ} \mathrm{C}$. IR (KBr) $v_{\max } 2931,2863,1455,1118,1097$, $733,695 \mathrm{~cm}^{-1}$. ${ }^{1} \mathrm{H} \mathrm{NMR}\left(\mathrm{CDCl}_{3}, 600 \mathrm{MHz},-20{ }^{\circ} \mathrm{C}\right.$, a 2:1 mixture of DOWN and UP conformers) $\delta 1.40-1.47(1 \mathrm{H}, \mathrm{m}, \mathrm{H}-17), 1.45-1.51(1 \mathrm{H}, \mathrm{m}, \mathrm{H}-16), 1.49-1.56(1 \mathrm{H}, \mathrm{m}$, H-3), 1.64-1.71 (2H, m, H-2), 1.70-1.75 (1H, m, H-17), 2.05 (1/3H, dd, $J=13.5,5.5$ $\left.\mathrm{Hz}, \mathrm{H}-9_{\mathrm{UP}}\right), 2.07-2.12\left(1 / 3 \mathrm{H}, \mathrm{m}, \mathrm{H}-6_{\mathrm{UP}}\right), 2.07-2.12\left(2 \times 2 / 3 \mathrm{H}, \mathrm{m}, \mathrm{H}-6_{\text {DOWN }}\right.$ and $\mathrm{H}-$ $3_{\text {DOWN }}$ ), 2.10-2.15 (1/3H, m, H-12 $\left.{ }_{\text {UP }}\right), 2.10-2.15$ (2x2/3H, m, H-12 $\left.{ }_{\text {DOWN }}\right), 2.14-2.18$ $(1 \mathrm{H}, \mathrm{m}, \mathrm{H}-16), 2.18-2.22\left(1 / 3 \mathrm{H}, \mathrm{m}, \mathrm{H}-3_{\mathrm{UP}}\right), 2.25(2 / 3 \mathrm{H}, \mathrm{dd}, J=12.5,5.5 \mathrm{~Hz}, \mathrm{H}-$ $\left.9_{\text {DOWN }}\right), 2.28\left(1 / 3 \mathrm{H}, \mathrm{dd}, J=14.0,5.0 \mathrm{~Hz}, \mathrm{H}-12_{\mathrm{UP}}\right), 2.75(2 / 3 \mathrm{H}$, ddd, $J=12.5,10.5$, $\left.9.5 \mathrm{~Hz}, \mathrm{H}-6_{\text {DOWN }}\right), 2.80$ (2/3H, ddd, $\left.J=12.5,9.5,9.5 \mathrm{~Hz}, \mathrm{H}-9_{\text {DOWN }}\right), 2.92-2.96$ (1/3H, m, H-9 $\left.{ }_{U P}\right), 2.94-2.98$ (1/3H, m, H-6 $\left.{ }_{U P}\right), 3.00(2 / 3 \mathrm{H}, \mathrm{dd}, J=9.5,9.5 \mathrm{~Hz}, \mathrm{H}-$ $\left.5_{\text {DOWN }}\right), 3.13\left(2 / 3 \mathrm{H}, \mathrm{dd}, J=9.5,9.5 \mathrm{~Hz}, \mathrm{H}-10_{\text {DOWN }}\right), 3.17(1 \mathrm{H}, \mathrm{ddd}, J=9.5,9.5,4.0$ Hz, H-15), 3.25 (1/3H, ddd, $J=10.5,10.0,4.5 \mathrm{~Hz}, \mathrm{H}-4 \mathrm{UP}), 3.31$ (1/3H, dd, $J=10.0$, $\left.5.5 \mathrm{~Hz}, \mathrm{H}-5_{\mathrm{UP}}\right), 3.32-3.38$ (1H, m, H-1), 3.33 (2/3H, dd, $J=9.5,3.5 \mathrm{~Hz}, \mathrm{H}-14_{\text {DOWN }}$ ), $3.40\left(1 / 3 \mathrm{H}, \mathrm{dd}, J=9.5,4.0 \mathrm{~Hz}, \mathrm{H}-14_{\mathrm{UP}}\right), 3.41(2 / 3 \mathrm{H}, \mathrm{ddd}, J=10.5,9.5,4.5 \mathrm{~Hz}, \mathrm{H}-$ $\left.4_{\text {DOWN }}\right), 3.46-3.49$ (2H, m, H-19), 3.48-3.52 (1/3H, m, H-13 $\left.{ }_{\mathrm{UP}}\right), 3.53-3.58$ (1/3H, m, H-10 $\left.{ }_{U P}\right), 3.57-3.61(1 \mathrm{H}, \mathrm{m}, \mathrm{H}-18), 3.71-3.74\left(1 / 3 \mathrm{H}, \mathrm{m}, \mathrm{H}-11_{\mathrm{UP}}\right), 3.71-3.74(2 / 3 \mathrm{H}, \mathrm{m}$, H-13 DOWN $), 3.82$ (2/3H, ddd, $\left.J=9.5,9.0,2.5 \mathrm{~Hz}, \mathrm{H}-11_{\text {DOWN }}\right), 3.86-3.92(1 \mathrm{H}, \mathrm{m}, \mathrm{H}-$ 1), $4.60\left(1 / 3 \mathrm{H}, \mathrm{d}, J=12.0 \mathrm{~Hz},-\mathrm{OCH}_{2} \mathrm{Ph}_{\mathrm{UP}}\right), 4.60\left(2 \times 2 / 3 \mathrm{H}, \mathrm{s},-\mathrm{OCH}_{2} \mathrm{Ph}_{\mathrm{DOWN}}\right), 4.60$ $\left(2 \times 1 / 3 \mathrm{H}, \mathrm{s},-\mathrm{OCH} \mathrm{Ph}_{\mathrm{UP}}\right), 4.61\left(2 / 3 \mathrm{H}, \mathrm{d}, J=12.0 \mathrm{~Hz},-\mathrm{OCH}_{2} \mathrm{Ph}_{\mathrm{DOWN}}\right), 4.68(1 / 3 \mathrm{H}, \mathrm{d}$, $\left.J=12.0 \mathrm{~Hz},-\mathrm{OCH}_{2} \mathrm{Ph}_{\mathrm{UP}}\right), 4.70\left(2 / 3 \mathrm{H}, \mathrm{d}, J=12.0 \mathrm{~Hz},-\mathrm{OCH}_{2} \mathrm{Ph}_{\text {DOWN }}\right), 5.71(2 / 3 \mathrm{H}$, ddd, $\left.J=11.0,9.5,5.5 \mathrm{~Hz}, \mathrm{H}-8_{\text {DOWN }}\right), 5.72(2 / 3 \mathrm{H}$, ddd, $J=11.0,10.5,5.5 \mathrm{~Hz}, \mathrm{H}-$ $\left.7_{\text {DOWN }}\right), 5.80\left(1 / 3 \mathrm{H}, \mathrm{ddd}, J=11.0,10.5,5.5 \mathrm{~Hz}, \mathrm{H}-8_{\mathrm{UP}}\right), 5.84(1 / 3 \mathrm{H}, \mathrm{ddd}, J=11.0$, $10.5,5.0 \mathrm{~Hz}, \mathrm{H}-7 \mathrm{UP}), 7.29-7.40\left(10 \mathrm{H}, \mathrm{m}\right.$, aromatic). ${ }^{13} \mathrm{C} \mathrm{NMR}\left(\mathrm{CDCl}_{3}, 150 \mathrm{MHz}\right.$, at $-20{ }^{\circ} \mathrm{C}$, a $2: 1$ mixture of DOWN and UP conformers) $\delta 25.59,25.90,27.36,27.48$, $29.40,29.69$, 30.14, 31.24, 31.35, 31.61, 32.33, 32.89, 33.96, 35.47, 38.97, 39.06, $67.98,68.36,70.72,71.01,72.35,73.12,76.13,76.40,77.85,78.32,78.44,79.57$, $80.78,81.48,82.48,82.77,86.16,86.49,86.55,86.63,87.40,127.33,127.37$, 
$127.40,127.54,127.61,127.91,128.12,128.24,128.34,128.94,137.84,138.28$, 138.34. ESI Q-TOF MS calcd for $\mathrm{C}_{33} \mathrm{H}_{42} \mathrm{NaO}_{6}[\mathrm{M}+\mathrm{Na}]^{+}$557.288, found 557.285. $[\alpha]_{D^{25}}+21.7^{\circ}\left(c 0.32, \mathrm{CHCl}_{3}\right)$. Anal. Calcd for $\mathrm{C}_{33} \mathrm{H}_{42} \mathrm{O}_{6}: \mathrm{C}, 74.13 ; \mathrm{H}, 7.92$. Found: C, 73.96; H, 8.03. 

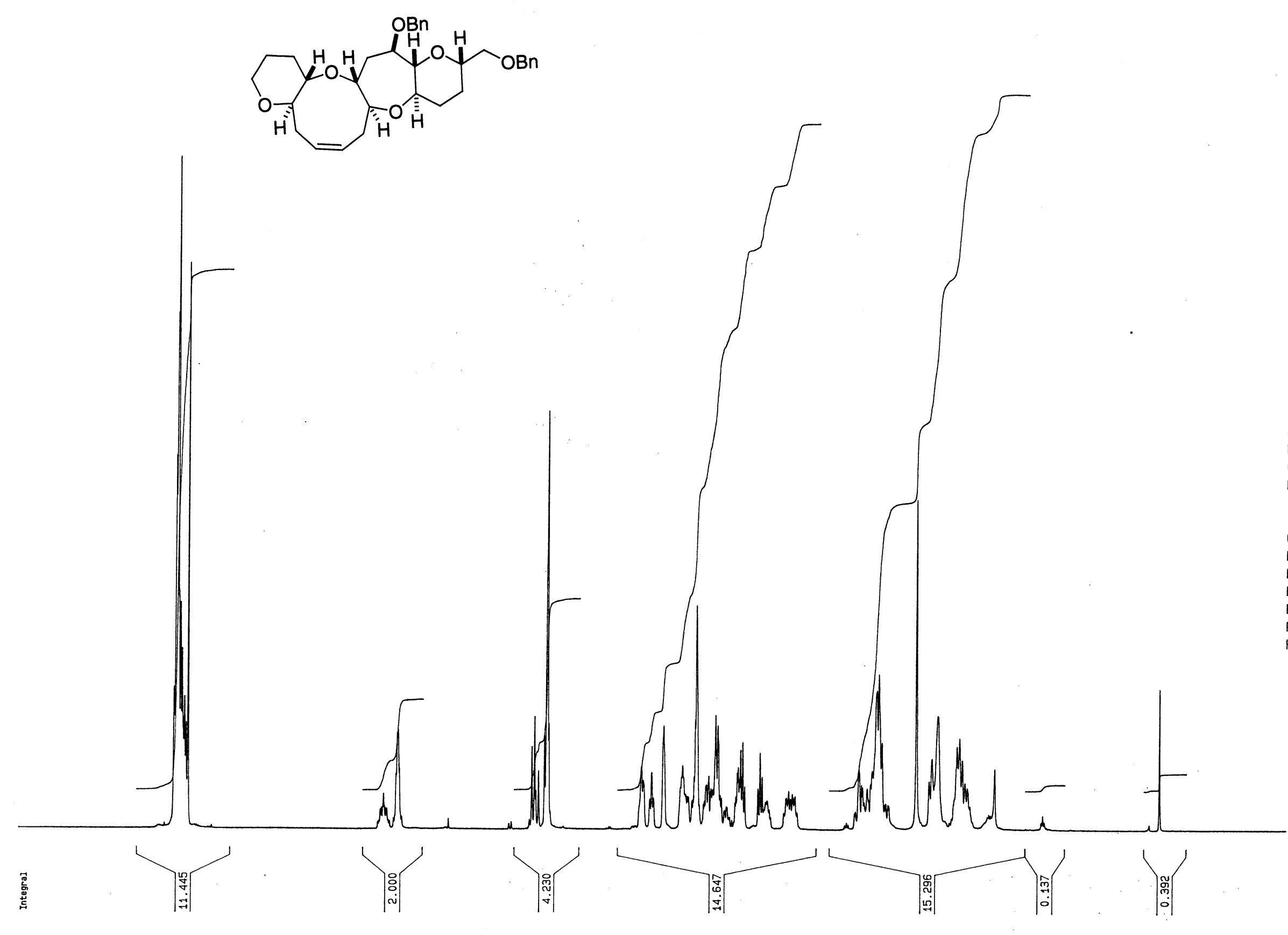

F2 - Acquisition Parateters

Date_ 1011023

$\begin{array}{lr}\text { Time } & 2.34\end{array}$

5 AMX2600

$5 \mathrm{~mm}$ Dual 19

PULPROG

290
32768

TD

SOLVEN

NS

SWH

FIDRES

RG

DW

F2

$\mathrm{SI}$

SF

SSB

LB

$G B$

$P C$

10 NMR plot parameters

Processing parameters $600.1364600 \mathrm{MHz}$ EM M

$$
\text { EM }
$$

$0.10 \mathrm{~Hz}$

0

$33.90 \mathrm{~cm}$ $8.500 \mathrm{ppm}$ $-1.000 \mathrm{ppm}$ $-600.14 \mathrm{~Hz}$ $0.024 \mathrm{~Hz}$ $0.28024 \mathrm{ppm} / \mathrm{cm}$ 